\title{
An Interval Intelligent-based Approach for Fault Detection and Modelling
}

\author{
Abbas Khosravi, Joaquim Armengol Llobet, Esteban R. Gelso
}

\begin{abstract}
Not considered in the analytical model of the plant, uncertainties always dramatically decrease the performance of the fault detection task in the practice. To cope better with this prevalent problem, in this paper we develop a methodology using Modal Interval Analysis which takes into account those uncertainties in the plant model. A fault detection method is developed based on this model which is quite robust to uncertainty and results in no false alarm. As soon as a fault is detected, an ANFIS model is trained in online to capture the major behavior of the occurred fault which can be used for fault accommodation. The simulation results understandably demonstrate the capability of the proposed method for accomplishing both tasks appropriately.
\end{abstract}

\section{INTRODUCTION}

Nowadays, fault detection and accommodation is a hot research line among different groups across the world. This is due to this fact that it is not only a real challenging problem from scientific point of view, but also industry insistently calls for more reliable, practical, self-reliant methods. Needless to say, such methods can bring more financial profit through decreasing the rate of system shut down for repair or avoiding serious destruction in different parts. Far more important, they raise the safety of the working environments and to a high degree prevent the occurrence of disastrous accidents for operators.

Hardware redundancy broadly used in 70 s for fault detection has its own drawbacks for being used in industry such as expensive implementation or high maintenance costs. Furthermore, in some cases its implementation is restricted or perhaps impossible because of some consideration related to weight and space [1].

Through screening the literature, we can classify all the analytical redundancy Fault Detection and Isolation (FDI) approaches in two generic schools: model-based and databased [1]. In model-based approaches, it is supposed that a quantitative or qualitative model of the underlying system is known. In a strike contrast, the data-based approaches need not such a model and they detect faults through exploring available data for finding some abnormalities in the behaviour of the system [1]. A comprehensive review of these approaches covering the method characteristics, advantages and drawbacks can be found in [2], [3], [4].

The most and actually foremost substantial deficiency of model-based approaches for FDI is their low performance when uncertainties are prevailing in the underlying system. In fact, not only uncertainties exist in the analytical model

A. Khosravi, J. Armengol and E. R. Gelso are with the Institut d'Informàtica i Aplicacions, Universitat de Girona, E-17071 Girona, Catalonia, Spain $\{$ khosravi, armengol, ergelso\}eeia.udg.es of the system owing some complicacy in modeling, but also they influentially mischievously impress measurements. A countless number of researchers have devoted a great deal of effort and time in last decades to deal with these uncertainties and confine their negative effects on fault detection and false alarm rates [5], [6], [7], [8], [9], [10].

In this paper we try to deal with this problem in a more practical fashion. We develop an interval model which is a set of models instead of one to take into account the majority of uncertainties in the underlying system. After that, a vigorous software package based on Modal Interval Analysis is developed which can robustly distinguish the occurrence of the fault through monitoring the difference between the measurements and the predicted values. As a part of fault accommodation procedure, then we model the occurred fault in a short time after its detection in online through employing the ANFIS capability for modelling of nonlinear systems.

Following the introduction, this paper has been organized as follows. The problem is stated in section II. The fundamental concepts of modal intervals and the proposed method for fault detection have been illustrated in section III. Section IV first concisely illustrates the ANFIS structure and then presents the proposed technique for fault modelling. Simulation results and conclusion constitute the final parts of this paper.

\section{Problem Overview}

Each analytical model of a system has some functions with some real-valued parameters which are not much accurate. This is due to this fact that modelling of all uncertainties in a system because of highly complicate interaction between different parts is roughly impossible. Furthermore, most of the parameters in real world plants are varying along the time aggravating the situation.

From a different angle, in practice, measurements are available instead of real outputs. Some inaccuracy always exists in measurements owing uncertainties prevailing in the measurement procedure. Malfunction of the Controller and imperfect operation of the fault detection process are results of such an uncertainty.

These two kinds of uncertainty are the most ruinous causes for impracticality or, at least, weakness of model-based FDI approaches in real world applications. Fig. 1 simply depicts how these uncertainties move into the plant. Finding a way for presenting them in the analytical model and confining their effects is the core motive for conducting this research. 


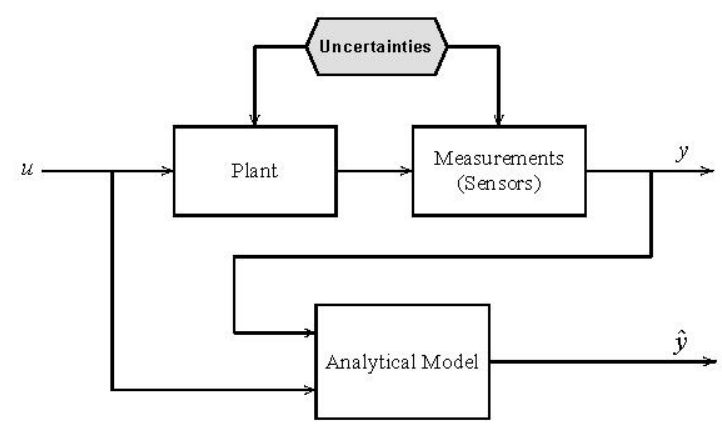

Fig. 1. Effect of uncertainties on system

\section{Fault Detection}

In this section we describe the proposed method for fault detection in some steps which makes its implementation more straightforward. It has been tried to put the idea of the fault detection in the simplest way and mathematical formulation has been presented wherever needed.

A simple but useful way for taking into account parameter uncertainties is to substitute the real-valued parameters with intervals. Applying this method results into a set of models instead of one. Calculation of the output evolution of such a model set, $y_{r}(t)$, generates a set of curves which are always between two bounds:

$$
Y_{r}(t)=\left[y_{r_{\min }}(t), y_{r_{\max }}(t)\right] .
$$

Consequently, an abnormal condition can be detected whenever

$$
y(t) \notin Y_{r}(t)
$$

where $y(t)$ is the real output of the system.

Measurement uncertainties cause

$$
y_{m}(t) \neq y(t) .
$$

This inaccuracy considerably increases the rate of false alarm; i.e. while real output is inside the computed range, $\left(y(t) \in Y_{r}(t)\right)$, the measurements are outside $\left(y_{m}(t) \notin\right.$ $Y_{r}(t)$ ).

One option for removing this inaccuracy is definition of interval measurement:

$$
y(t) \in Y_{m}(t)
$$

where

$$
Y_{m}(t)=\left[y_{m}(t)\left(1-e_{r}\right)-e_{a}, y_{m}(t)\left(1-e_{r}\right)+e_{a}\right] .
$$

$e_{r}$ and $e_{a}$ correspond to the relative and absolute errors respectively.

A fault alarm will be set on whenever

$$
Y_{m}(t) \cap Y_{r}(t)=\emptyset .
$$

Computation of $Y_{r}(t)$ is a real time-consuming task in a multi-dimensional space and should be repeated in each step. Augmentation of the computation mass is another problem that becomes serious as time goes ahead [11].
To decrease the computation mass, we compute an external estimate of $Y_{r}(t)$ and substitute it in (6). It results in

$$
Y_{m}(t) \cap Y_{\text {rex }}(t)=\emptyset,
$$

Using monotonic inclusion property of internal arithmetic which says if $\boldsymbol{X} \subset \boldsymbol{Y}$, then $F(\boldsymbol{X}) \subset F(\boldsymbol{Y})$, we can compute $Y_{\text {rex }}(t)$ easily [12]. Interval arithmetic operations are the natural extension of a rational function

$$
R_{f}(\boldsymbol{X}) \subseteq f R(\boldsymbol{X}) .
$$

$f R(\boldsymbol{X})$ and $R_{f}(\boldsymbol{X})$ are natural extension and the range of the function $f$ respectively.

Through splitting $\boldsymbol{X}, \boldsymbol{X}_{1} \cup \boldsymbol{X}_{2}=\boldsymbol{X}$, we can rewrite (8)

$$
R_{f}(\boldsymbol{X}) \subseteq f R\left(\boldsymbol{X}_{1}\right) \cup f R\left(\boldsymbol{X}_{2}\right) \subseteq f R(\boldsymbol{X}),
$$

To put an end to the splitting algorithm in (9), we introduce an internal estimate, $Y_{\text {rin }}(t) \subseteq Y_{r}(t)$. If $Y_{m}(t) \cap Y_{\text {rin }}(t) \neq \emptyset$, then, logically we can expect that $Y_{m}(t) \cap Y_{r}(t) \neq \emptyset$. It means that there is no fault detection and we should stop splitting.

So far, we have obtained the same results of fault detection using (1) with quite less computation mass and without false alarm rate. Branch-and-bound algorithms [13] can be employed for iterative computation of $Y_{\text {rin }}(t)$ and $Y_{\text {rex }}(t)$. As a conspicuous property, they eliminate larger subspaces more quickly than do the other techniques [14].

To reduce the computation mass more, we skillfully introduce the Multiple Sliding Time Window (MSTW) technique for estimation of the future states using initial states and inputs. In this fashion, we predict the output from an initial value between $0<t_{i}<t$, i.e., $Y_{r}\left(t \mid t_{i}\right)$ where $t-t_{i}=w$. $w$ is named time window and fixing it successfully confines the computation attempt for obtaining a variable prediction. Apparently, a longer time window calls for more effort. In simulation, $w$ starts with the smallest value and goes up to a time that it gets its maximum possible value or the occurred fault is detected. The procedure of the proposed method understandably has been illustrated in Fig. 2 .

Although the developed procedure is simple, it significantly declines the computation mass for fault detection. The most important characteristic of the proposed method for fault detection is its robustness. It no doubt guarantees that there is no false alarm provided that the interval model and measurements adequately represent the system and the true values of the variables. The last but not the least, the simultaneous use of several window lengths not only confines the computation effort, but also reduces the rate of missed alarm.

\section{FAUlt Modelling}

In the previous section we described how we detect the occurred fault while the false alarm rate is minimized. In the next stage and for fault accommodation, we start to model the detected fault through adjusting parameters of an ANFIS model based on available measurements. 


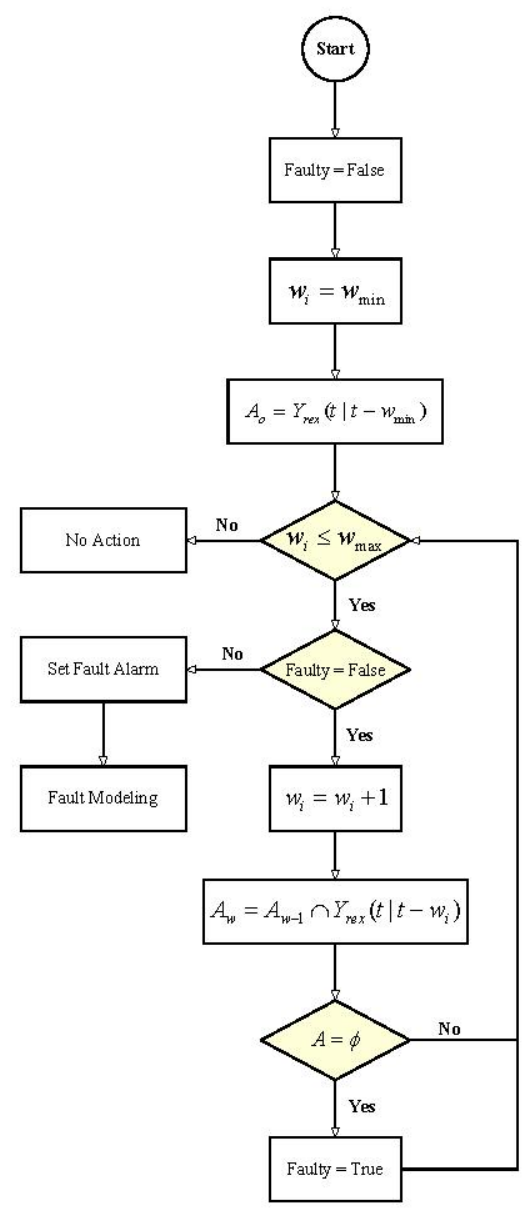

Fig. 2. MSTW algorithm

and $c_{k}$ are consequent parameters which should be trained.

As shown in Fig. 3, the ANFIS structure contains five layers where two of them are adaptive and the rest are fixed. In fact, ANFIS is a feedforward neural network which implements a fuzzy inference system. The following points about these layers are noteworthy, helping to find a good sight to ANFIS structure and output generation.

Nodes in layer 1 are membership functions generating the degree of membership of inputs. In Fig. 3, $A_{i}$ and $B_{i}$ are presenting membership functions for each input. Oftentimes they are gaussian functions with two or three parameters named premise parameters. Having tunable parameters in nodes is the reason for calling this layer a tunable/adaptive layer.

Each node in the second layer multiplies the degree of membership functions and generates firing strength for each rule:

$$
w_{k}=\mu_{A_{i}}\left(x_{1}\right) \mu_{B_{j}}\left(x_{2}\right), i, j=1,2,3, k=1, \ldots 9
$$

Apparently, this layer is a fixed layer without any parameter to be tuned.

Like the second layer, the third layer is a fixed layer and inputs are normalized in each node:

$$
\bar{w}_{k}=\frac{w_{k}}{\sum_{i=1}^{9} w_{i}}, k=1, \ldots 9
$$

The fourth layer is a realization of the then-part of each fuzzy rule in ANFIS structure. A linear polynomial of inputs and normalized firing strength are multiplied in each node of this layer:

$$
Y_{k}=\bar{w}_{k}\left(a_{k} x_{1}+b_{k} x_{2}+c_{k}\right), k=1, \ldots 9
$$

As mentioned before, we refer to parameters of this layer

\section{A. ANFIS}

In this part we introduce the structure of ANFIS with some mathematical points useful for explanation of our method.

ANFIS is an excellent extension of fuzzy inference system which utilizes the learning capabilities of neural networks. Both fuzzy inference system (FIS) and neural network (NN) have their own particular disadvantages. In literature, many striking applications of fuzzy inference system and neural network have been reported in diverse areas.

Obtaining good results from FIS needs devoting considerable times and efforts for proper selection of number, kind, and parameters of membership functions. While parameters in NNs are quickly adjusted through a learning method, they do not use human's knowledge in their structure or training stage and, therefore, lack transparency.

An ANFIS system structure with two inputs and one output has been pictured in Fig. 3. In this presentation, there are three membership functions for each input which results in 9 rules like this: If $X_{1}$ is $A_{i}$ and $X_{2}$ is $B_{j}$, then

$$
Y_{1}=a_{k} x_{1}+b_{k} x_{2}+c_{k}
$$

where $i, j=1,2,3$ and $k=1,2, \ldots 9$. In (10), $x_{1}$ and $x_{2}$ are inputs to ANFIS and $Y_{i}$ is output of each rule. $a_{k}, b_{k}$ as consequent parameters which introduce this layer as a tunable/adaptive one.

Finally, the fifth layer is a fixed one generating the overall output of ANFIS:

$$
Y=\sum_{k=1}^{9} \bar{w}_{k} Y_{k}=\sum_{k=1}^{9} \bar{w}_{k}\left(a_{k} x_{1}+b_{k} x_{2}+c_{k}\right)
$$

\section{B. Fault Modelling}

The proposed method for fault modelling in closed-loop systems has been schematically illustrated in Fig. 4.

We develop an observer using the analytical model of the system to tune the parameters of the ANFIS model. This observer which is an appreciable combination of classical and intelligent models can be presented like this:

$$
\dot{\hat{y}}=F(y, u)+\hat{f}(y, u)+G(\hat{y}-y)
$$

where $y$ and $\hat{y}$ are real output and observer output respectively. In $(15), F($.$) is the analytical model of the underlying$ plant and $\hat{f}(\cdot)$ is the ANFIS model of the fault which should be tuned [15], [16], [17], [18]. Stability of the observer can be guaranteed by choosing $G$ as a positive definite matrix. Parameter values in $F(\cdot)$, plant model, take their nominal 


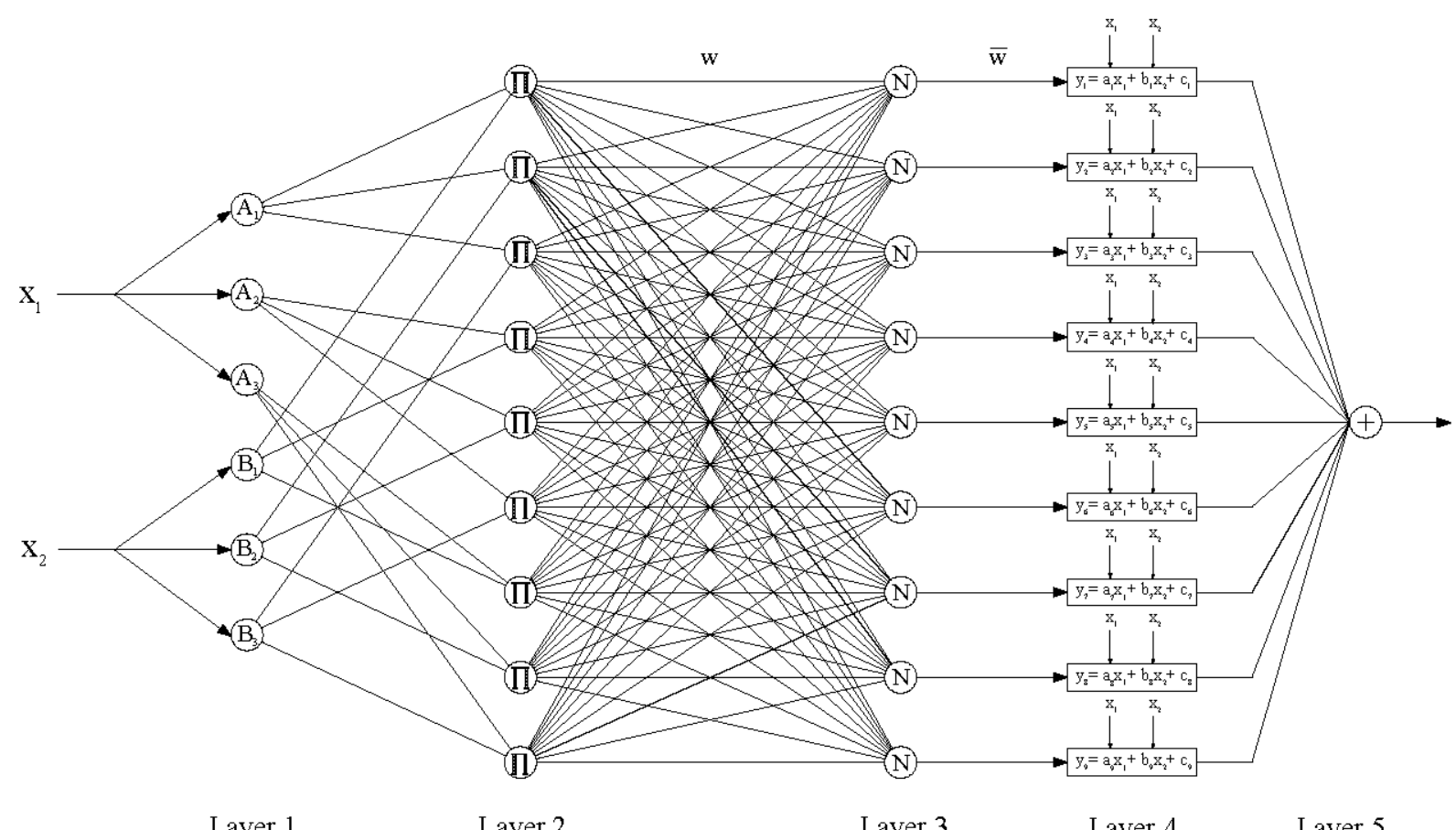

$\begin{array}{llll}\text { Layer } 1 & \text { Layer } 2 & \text { Layer } 3 & \text { Layer } 4\end{array}$

Fig. 3. ANFIS structure

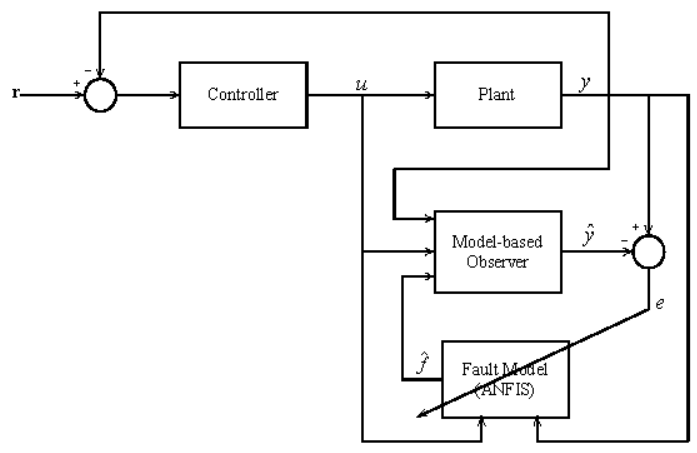

Fig. 4. Schematic of fault modelling technique

values which are the best available choice regarding to our information of the plant.

Using well-known back-propagation learning method, both premise and consequent parameters of the ANFIS model can be adjusted. But, oftentimes, it is not necessary that we spend our time for precise tune of premise parameters. This is due to this fact that they have a less impressive effect on ANFIS output than consequent parameter. Based on this and through proper selection of number and kind of inputs and their membership functions, we can reach satisfying results through just modifying consequent parameters. This significantly reduces the computation mass and increases the speed of fault modelling procedure. Doing this, expectedly, inevitably causes some slight impression in modelling. Nonetheless, advantages still considerably outweigh possible disadvantages.

ANFIS consequent parameters, $\theta_{\hat{f}}(\cdot)$, are adjusted in online. Whenever a new measurement is available, we adapt them as follows

$$
\theta_{\hat{f}}(t)=\theta_{\hat{f}}(t-1)-\lambda \frac{\partial J(t)}{\partial \theta_{\hat{f}}(t-1)}
$$

where $\lambda$ is the learning rate.

As shown in Fig. 4, the objective functional which should be minimized here is

$$
J=\frac{1}{2}(y-\hat{y})^{2} .
$$

If we define estimation error as

$$
e=\hat{y}-y,
$$

term $\frac{\partial J(t)}{\partial \theta_{\hat{f}}(t-1)}$ in (16) can be mathematically expanded using partial derivative rule

$$
\frac{\partial J}{\partial \theta_{\hat{f}}}=\frac{\partial J}{\partial e} \frac{\partial e}{\partial \theta_{\hat{f}}}=e \frac{\partial e}{\partial \theta_{\hat{f}}} .
$$

To compute $\frac{\partial e}{\partial \theta_{\hat{f}}}$, we need to obtain dynamic of the estimation error. Subtracting (15) from the intact analytical model of the system gives

$$
\dot{e}=\dot{\hat{y}}-\dot{y}=G(\hat{y}-y)+\hat{f}(y, u)=G e+\hat{f}(y, u) .
$$

This shows that after disappearing initial value effect, estimation error only depends on the occurred fault. Therefore, we can rewrite (19)

$$
\frac{\partial J}{\partial \theta_{\hat{f}}}=e \frac{\partial \hat{f}}{\partial \theta_{\hat{f}}}
$$


which its calculation is straightforward based on specific structure of ANFIS as presented in the previous subsection. This completes our discussion for fault modelling.

A characteristic more important than implementation ease of the proposed method is its capability to cover different occurred faults in sensors, actuators, and plant components without any concern about complexity of faults or even having time-variant dynamic. We will address this striking property in future works.

\section{Simulation Results}

In this section, we implement the proposed method in the previous sections on a nonlinear plant. The considered plant on this research work is a tank that has a controlled valve in its output. A PI controller controls the valve based on measurement and reference signal of the tank liquid level. Fig. 5 schematically simply shows different parts of the underlying system.

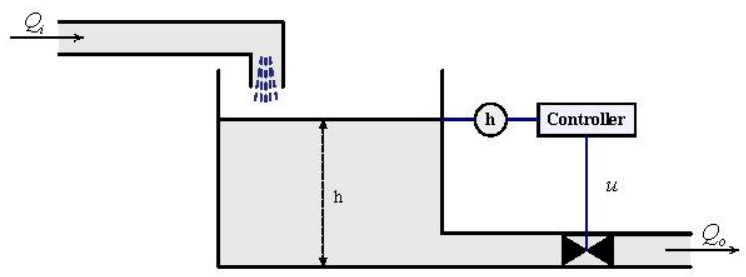

Fig. 5. The controlled tank system

Mathematically, we can present variation of the tank level, $h(\cdot)$, of this system as follows

$$
A \dot{h}(t)=Q_{i}(t)-Q_{o}(t)=Q_{i}(t)-\gamma u(t) \sqrt{h(t)}
$$

in which $Q_{i}(\cdot)$ and $Q_{o}(\cdot)$ are input and output flows respectively. $u($.$) is PI controlling signal and \gamma$ is a physical parameter.

Both $\gamma$ and the level measurement have their own uncertainties. For $\gamma$ we consider about $\pm 10 \%$ variation around its nominal value as uncertainty in physical parameters. Also, uncertainty in measurement of $h(\cdot)$ is considered as $h(\cdot)(1 \pm 0.05)$.

For fault detection stage, we develop a software package named SQualTrack (Semi-Qualitative Tracking) which appropriately executes what was formerly explained in section III. We introduce plant model in this software with the considered uncertainties in the plant parameters and measurements.

Because of the slow behaviour of the underlying plant, sample time for measurement of output was fixed at $1 \mathrm{~s}$ without any concern about missing any noticeable deviation. In simulation, tank level should follow a reference signal which varies between $2.7 \mathrm{~m}$ and $3.3 \mathrm{~m}$. To examine its capability of fault detection, a leakage fault was deliberately mathematically generated in the system at $370 \mathrm{~s}$. Needless to say, the severity of this fault is directly related to the square root of $h(\cdot)$. Plots of internal, external, and measurement envelopes generated by SQualTrack as well as fault alarm have been presented in Fig. 6. Void intersection of external envelope with measurement envelope for the first time occurs is $375 \mathrm{~s}$, just five seconds after fault occurrence. Fault occurrence and detection times have been clearly marked in Figs. 6(a) and 6(b) respectively.
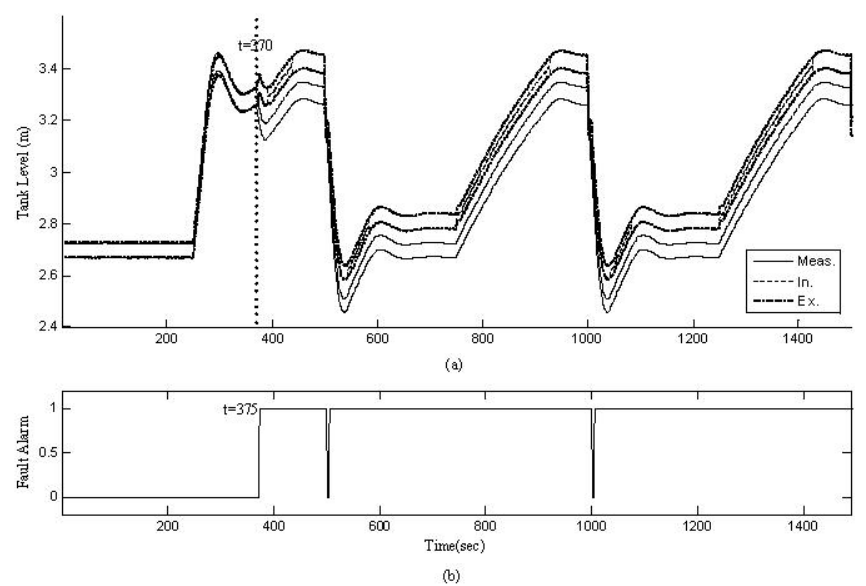

Fig. 6. (a) External, internal, and measurements envelopes generated in SQualTrack, (b) Fault alarm

It's noteworthy to mention that this fault detection simulation highlights a striking property of the proposed method. As shown in Fig. 6(b), there is no false alarm before occurrence of the fault. In the other word, while the analytical model of the system and measurement have some kinds of uncertainty, SQualTrack is quite robust against them and doesn't consider them as a fault. Furthermore, after fault occurrence, it based on computed void intersection of envelopes immediately detects occurrence of the fault and continuously keeps the fault alarm ON throughout the simulation except for some negligible instants.

Simulated leakage fault depends on the volume of liquid inside tank. Taking into account this point, we consider sensor measurement and its previous values as inputs of ANFIS model. In this simulation, ANFIS has two inputs which discourse universe of each one is covered with three Gaussian membership functions. This structure of ANFIS includes 27 consequent parameters distributed in 9 if-then rules. As mentioned in previous section, in contrast with consequent parameters, 12 premise parameters are appropriately chosen and fixed which fulfill the required precession in our simulations.

Adjusting ANFIS model parameters starts at $375 \mathrm{~s}$, the instant that we detect fault, and continues approximately for $280 \mathrm{~s}$. Fig. 7(a) displays the tank reference and actual levels in normal and faulty situations. Tank level deviates from its nominal behaviour at $370 \mathrm{~s}$ and the controller attempts for revitalizing the system to its normal behaviour are approximately abortive. The actual and estimated fault values have been depicted in Fig. 7(b). Obviously after the training time, ANFIS output matches the simulated fault 
acceptably which implies that the fault modelling task has been done well.
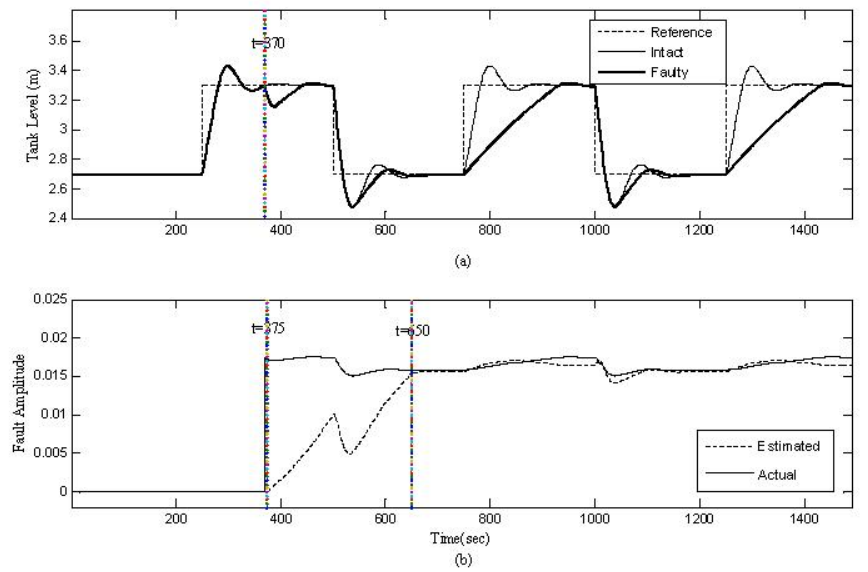

Fig. 7. (a) Reference and actual values of tank level in normal and faulty situations, (b) Actual and estimated fault

It's true that there are some errors in modelling, but they are so slight that we can safely disregard them. Also, these small differences can be treated well through more accurate manual tuning of premise parameters. Needless to say, hiring more membership functions for inputs or increasing the number of inputs can be exploited to enhance fault modelling precision if more needed, although the obtained results in this simulation acceptably sounds satisfying.

We run many simulations to examine the capability of the proposed method in both detection and modelling stages. Reference input, the occurrence time of the fault and its severity were changed for covering different cases. The obtained results in these simulations are very similar to the presented results in this section which strengthen powerfulness of the developed methods.

\section{CONCLUSION}

In this paper we addressed FDI difficulties arising from presence of uncertainties in the analytical model of the plant and in measurements. Using modal interval theory, a vigorously robust technique for fault detection was developed. This technique is based on an analytical model which its parameters are intervals instead of fixed values. After detection of a fault, we modelled it in online using ANFIS a short time after its detection. The capability of the proposed method for FDI was properly shown through its implementation on a closed loop nonlinear system.

The performance of the proposed method can be still enhanced in both detection and modelling stages. Using the fault model for modification of controlling signal is an interesting issue for completing fault accommodation task. Moreover, it is possible that we identify and model occurred faults in sensors and actuators under the proposed circumstance with some modification. Both will be addressed in future research works.

\section{ACKNOWLEDGMENTS}

This work has been funded by the European Union (European Regional Development Fund) and the Spanish Government (Plan Nacional de Investigación Científica, Desarrollo e Innovación Tecnológica, Ministerio de Ciencia y Tecnología) through the co-ordinated research projects MCYT DPI2003-07146-C02-02 and DPI2004-07167-C02-02. The authors gratefully acknowledge the helpful contribution of Ms Reihaneh Naghavizadeh for drawing some pictures of this paper.

\section{REFERENCES}

[1] R. J. Patton, P. M. Frank, and R. N. Clark, Fault Diagnosis in Dynamic Systems, Theory and Application. Britain: Prentice Hall, 1989.

[2] V. Venkatasubramanian, R. Rengaswamy, K. Yin, and S. N. Kavuri, "A review of process fault detection and diagnosis: Part I: Quantitative model-based methods," Computers and Chemical Engineering, vol. 27, pp. 293-311, 2003.

[3] - "A review of process fault detection and diagnosis: Part II: Qualitative models and search strategies," Computers and Chemical Engineering, vol. 27, pp. 313-326, 2003.

[4] - "A review of process fault detection and diagnosis: Part III: Process history based methods," Computers and Chemical Engineering, vol. 27, pp. 327-346, 2003.

[5] W. E. Dixon, I. D. Walker, D. M. Dawson, and J. P. Hartranft, "Fault detection for robot manipulators with parametric uncertainty: A prediction error based approach," in 2000 IEEE International Conference on Robotics \& Automation, 2000, pp. 3628-3634.

[6] L.-C. C. Shen and P.-L. Shao-Kung Hsu, "Robust fault detection and isolation with unstructured uncertainty using eigenstructure assignment," in AIAA (American Institute of Aeronautics and Astronautics) Guidance, Navigation and Control Conference. Technical Papers. Pt. 3 (A95-39609 10-63), 1995, pp. 1861-1870.

[7] A. Johansson and T. Norlander, "Parametric uncertainty in sensor fault detection for a turbofan jet engine," in 42nd IEEE CDC, 2003, pp. 1950-1955.

[8] M. Bask, "Dynamic threshold generators for robust fault detection," Ph.D. dissertation, Control Eng. Group, Dept. of Comp. Science and Elec. Eng., Luleå University of Technology. http://epubl.1tu. se/1402-1544/2005/11/LTU-DT0511-SE.pdf, 2005.

[9] Z. Han, W. Li, and S. L. Shah, "Fault detection and isolation in the presence of process uncertainties," Control Eng. Practice, vol. 13, pp. $587-599,2005$.

[10] P. M. Frank, "Handling modelling uncertainty in fault detection and isolation systems," in 9th Int. Conf. IPMU, 2002, pp. 1729-1749.

[11] J. Armengol, J. Vehí, L. Travé-Massuyes, and M. A. Sainz, "Interval model-based fault detection using multiple sliding time windows," in 4th IFAC Symposium on Fault Detection, Supervision and Safety for Technical Processes SAFEPROCESS 2000. Budapest, Hungary, 2000, pp. 168-173.

[12] R. E. Moore, Interval analysis. Prentice-Hall, 1966.

[13] P. Herrero, M. Á. Sainz, J. Vehí, and L. Jaulin, "Quantified set inversion algorithm with applications to control," Reliable Computing, vol. 11, no. 5, pp. 369-382, 2005.

[14] J. Armengol, J. Vehí, L. Travé-Massuyès, and M. Á. Sainz, "Application of modal intervals to the generation of error-bounded envelopes," Reliable Computing, vol. 7, no. 2, pp. 171-185, April 2001.

[15] J.-S. R. Jang, "ANFIS: Adaptive-network-based fuzzy inference systems," IEEE Transactions on Systems, Man and Cybernetics, vol. 23 , no. 3, pp. 665-685, 1993.

[16] L. Wang, A Course in Fuzzy System and Control. Prentice Hall, 1997.

[17] J. J. Buckley and T. Feuring, Fuzzy and Neural: Interactions and Applications, ser. Studies in Fuzziness and Soft Computing. Heidelberg, Germany: Physica-Verlag, 1999.

[18] H. Nguyuen, N. Prasad, C. Walker, and E. Walker, A First Course in Fuzzy and Neural Control. Chapman \& Hall/CRC, 2003. 